\title{
Research on the Integration of Traditional Arts and Crafts into the Design of Modern Cultural and Creative Products
}

\begin{abstract}
Lan Jin
Visual Arts, Shanghai Art \& Design Academy, Shanghai,201800, China

Email: 1454664183@qq.com

Abstract

Traditional arts and crafts, mostly made by hand, represents the wisdom of laboring people over the generations. In modern design, however, the purely handmade way will lead to low efficiency and thus the inability to meet the current large-scale market demand. Many crafts are on the edge of failing to be handed down from past generations. The cultural and creative industries have played a critical role in the current economic development of the local regional economy. In particular, with the rapid development of urban cultural and creative industries in design and tourism, increasing attention has been paid to the development of modern cultural and creative product design. This paper focuses on the integration of traditional arts and crafts into the design of cultural and creative products, and the acquisition of new vitality through mutual integration. This paper elaborates on five strategies, that is, the refinement of elements in traditional arts and crafts will enhance the connotation of cultural and creative products; the rational use of traditional arts and crafts will increase the value of cultural and creative products; the style orientation of traditional arts and crafts will strengthen the personality of cultural and creative products; the functional development of traditional arts and crafts will increase the quality of cultural and creative products; the application of science and technology brings cultural and creative products with new value; and the branding of traditional arts and crafts will enhance the competitiveness of cultural and creative products.
\end{abstract}

Keywords: traditional crafts, cultural and creative products, design, integration

\section{INTRODUCTION}

Cultural and creative products are generally national and ethnic in nature, whose design includes various influences such as humanities, environment, and politics. On the strength of the development of science and technology and the globalization of economy over the recent years, the design industry of cultural and creative products has developed rapidly and even tuned into the economic pillar industry of some countries. As China is a country with a time-honored history and culture, Chinese arts and crafts have been inherited for a long time, so the introduction of traditional arts and crafts into modern design, especially in the design of cultural and creative products, has been even more meaningful.

As one of traditional Chinese cultures, traditional arts and crafts are unique artistic features formed following a long-standing historical development. When integrated into the design of cultural and creative products, they make the products more culturally rich, thus playing an overall enhancement effect.

\section{REFINING ELEMENTS TO ENHANCE THE CONNOTATION OF CULTURAL AND CREATIVE PRODUCTS}

There are a lot of humanities and history and various traditional crafts in each region that can be explored. Summarizing and refining the information and elements that are meaningful to cultural and creative design as well as using them reasonably in the design can enhance the connotation of the design of cultural and creative products. Prior to the development stage of the design of creative products, the failure to conduct in-depth research on the cultural background, thus causing a poor understanding of culture, will make the design superficial. Besides, induction and refinement to obtain a large amount of relevant information play a key role in 
the integration of traditional culture and modern design. Improving the rationality and logic of the combination of modern design language with handicrafts, and integrating refined arts and crafts into the cultural and creative design products can avoid the monolithic nature of the current cultural and creative products on the market.

China with its time-honored history always has some inexhaustible artistic inspiration to explore. You can adopt some classical historical stories, or find a lot of materials in the museum as artistic inspiration. For example, some instruments in local museums feature beautiful shapes and smooth and unique lines. Cultural and creative products can borrow these styles with sources and origins to make the product style more obvious. Then, we form our own unique product design that is distinct from other countries or nationalities. Product designers are also required to improve their cultivation and professionalism. With good cross-border thinking skills, they are expected to devote themselves to designing cultural and creative products that meet the needs of audiences of all ages (Figure 1.), while enabling the aesthetics of the public to be enhanced, and thus gaining the satisfaction of spiritual needs. By extracting elements from the design of traditional arts and crafts, redesigning the elements and applying them reasonably, the design of cultural and creative products shows new vitality. The cultural connotation is thus improved, and traditional arts and crafts are developed lastingly.

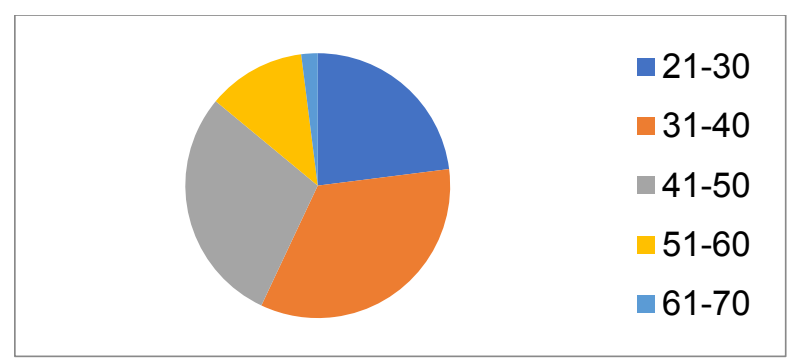

Figure 1 Age of Consumers

\section{RATIONAL USE TO INCREASE THE VALUE OF CULTURAL AND CREATIVE PRODUCTS}

At present, the commercial value of cultural and creative products has not been fully reflected. It's mainly because that the elements that can give it potential value have not been integrated. For example, many jewelry designs of international big brands are products combined with traditional crafts, and their sales are very considerable every time new products are promoted. China's traditional arts and crafts are very rich in variety. On the basis of a long evolution, each region has formed its own characteristics of arts and crafts, with many types including ceramic crafts, cloisonné crafts, wood carving, embroidery, weaving, metalworking, silk pinching crafts, jade carving crafts, etc. Each has its own special, visualized spiritual charm of aesthetics and values derived from the local arts and crafts. Nowadays, many local arts and crafts products do not really use traditional arts and crafts techniques, but simply paste and copy some tradition patterns without the necessary craft techniques. The proper use of arts and crafts is an issue that designers need to consider. The design of cultural and creative products should not only meet the scale of production, but also integrate traditional craft techniques, for example, the local use of craft techniques will be very effective. The embroidery, one of intangible cultural heritage, can be integrated into T-shirts or cloth material products. In particular, embroidered graphics can be embellished in collars, cuffs and other places. The embroidery not only makes a difference, but also brings a new meaning to the auspicious symbolism. The handmade embroidery is full of warmth, which makes the product more humane from the psychological point of view, and makes the product more valuable while inheriting it. Therefore, embroidery crafts are reasonably used in the design of cultural and creative products. (Figure 2.)

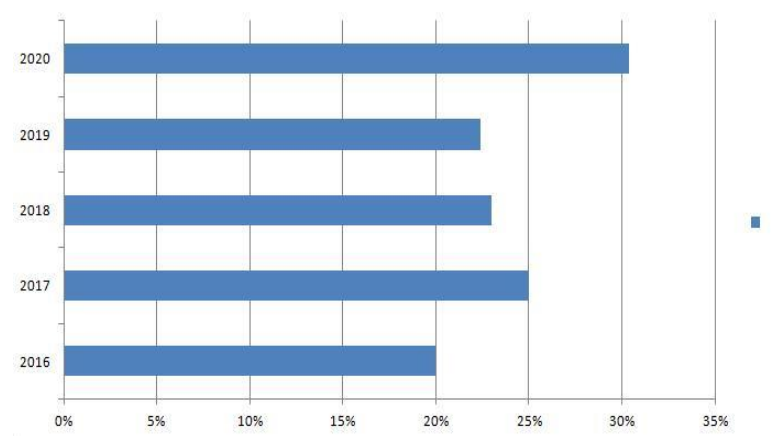

Figure 2 Growth of Sales of Cultural and Creative Products from 2016 to 2020

\section{STYLE POSITIONING TO STRENGTHEN THE PERSONALITY OF CULTURAL AND CREATIVE PRODUCTS}

People's aesthetic diversity makes the design of cultural and creative products in the need of being diversified and rich in personality. There is a design style of mild modeling lines; or a slightly processed rugged one, or simple modeling, etc. The language of traditional crafts is rich, and the design style can only be integrated with modern design after refinement. The design style is the core of the product design, so the mood it creates is of great significance. There are many aspects of style such as color style, styling style, etc. (Figure 3.) 


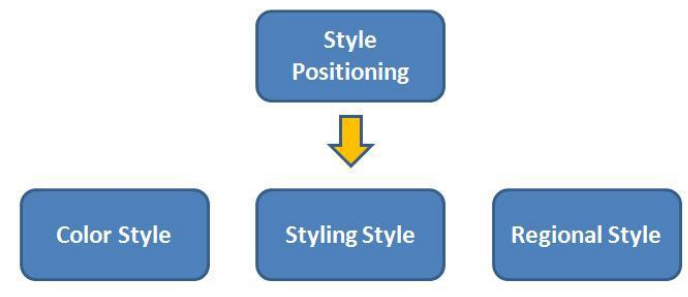

Figure 3 Style Positioning of Cultural and Creative Product Design

\subsection{Positioning of Color Style}

The use of color represents one's wisdom. The color application of traditional crafts is polarized. The one is more elegant and original, creating a primitive and simple style, and thus the cultural and creative products will basically also present a quiet and far-reaching design style; the other is a symbol of people's good wishes of warm color scheme, especially when the cultural products integrate the local unique colors to design products with local characteristics. Chinese red is the national color of China, and there are many cultural and creative products designed with red color. Each nationality and region also has its unique color scheme, which is a color application system that has evolved over a long period of time and distinguishes itself from other regions after the long-term use. For example, if we compare the web interfaces of different countries and regions, we can see that their colors differ significantly. Therefore, if we use these distinctive colors in the design of cultural and creative products, we can certainly enhance our own cultural pride. In addition to the recognized Chinese red, in fact, the "red" in ancient traditional arts and crafts are divided into many kinds; the ancients can identify carmine red, cinnabar, etc. In terms of "yellow", there are rattan yellow, light yellow, arth yellow, etc. The same goes with "green"; the Chinese ancients can identify blackish green, dark green, peacock green, spearmint, etc., most of which are from the color of minerals, long used in arts and crafts products. These color elements represent either country's colors or national regions [1]. Designers can choose different color styles to fit the product. The traditional craft color scheme must show its long-lasting charm in the context of the new era.

\subsection{Positioning of Styling style}

Styling design directly positions the style of cultural and creative products. The minimalist style with concise modeling language and strict and smooth lines is the design originally pursued in China. For example, the furniture design of the Ming Dynasty is concise in outline, moderate in proportion, and mainly based on lines. It can be used for reference in modern product design. The combination of simplicity and elegance will increase the overall visual effect of cultural and creative products. Another example is the simple teapot design, which still shows a sense of simplicity when used in today's design. There are a variety of styles, and there is an admiration for minimalism in oriental culture. This aesthetic can be reflected in the concise product lines, simple shapes and the use of original materials. By virtue of the removal of non-essential decorative elements, and the use of elegant and simple colors, the design of the product exudes a low-key, astringent, and classic quality that is worthy of playfulness. With the improvement of social development and material culture, there are more and more items, so these products will appear in different combinations and visual effects in the same space. Some people desire a sense of unity, and series design is thus born; while others will sometimes pursue individuality, where various styles of cultural and creative products are put together in a dazzling array. Exquisite craftsmanship and decorative style can make the product show a modern feeling of fashion, complexity and elegance. We see some round shapes representing complete abundance, and then with complementary or clashing color designs we can see a sense of Chinese cultural richness.

\subsection{Positioning of Regional Style}

In terms of large regions, the auspicious visual culture in Chinese traditional culture is unique, and the auspicious culture in the form, shape, color expresses the spiritual connotation in the Chinese traditional visual elements. In addition, the style of each region is also different. Many regions are now designed with strong folk culture. The content is rich in meaning, integrated in the product modeling, making it more auspicious and pleasant. This implicit function, in line with consumer psychology, may also be a very important concept of traditional Chinese culture to modern product design. Regions naturally have local unique materials. For example, some regions produce bamboo, and their cultural and creative products are developed and produced with bamboo as the material. Naturalistic style is to use natural materials as the main material, and take its shape, texture, texture, pattern that expresses nature, and then produce products with a little modification. Natural objects, with a sense of eternity and elegance without trace, are gifts from nature. The natural materials possess a unique beauty that emanates from the natural texture. Each region's cultural and creative products will have different materials, shapes, and details, making the commonplace materials into regional characteristic artworks, which, in addition to people's demand for functionality, can also build cultural and creative products with great regional cultural characteristics, providing different beauty and collection value. 


\section{FUNCTIONAL DEVELOPMENT TO INCREASE THE QUALITY OF CULTURAL AND CREATIVE PRODUCTS}

Cultural and creative products are mainly culture-led products transformed from creativity. However, now the practicality of cultural and creative products, that is, the development of functions, has become very essential. The reason is that most of the products sold in the market are basic functional products such as refrigerator stickers, folders, key rings and other small commodities. The need to study and develop the functionalities of creative products has become the key to the problem.

The cultural and creative products are transited from products with low price materials and low cost to such ones with practicability and even value preservation functions, investing themselves with inheritance functions. The integration of arts and crafts makes it possible for cultural and creative products to have the function of inheritance. Cultural and creative products should first meet the needs of the public, and it is reasonable to develop them at different levels. For example, a series of online celebrity products developed in the past few years were less expensive since the use of simple materials, which indeed play a role in promotion, but how to improve sales after market saturation? Only by printing and pasting and copying simple graphics, what may be produced is too simple, whose connotation value is far from enough. Therefore, it is necessary to classify the cultural and creative products and clarify whether they are consumables or long-term products, which are different in terms of design and materials used. In view of people's aesthetic and diversified needs, we need to find more hot spots to convert into cultural and creative product, and the product design also needs to be upgraded in place of those generalized designs. Besides, the development of the use function is imperative. For example, the White Rabbit, on the basis of the original people's preference for its candy, develops cultural and creative products with practical functions, such as umbrellas and bags. However, there comes a time when the refrigerator stickers are full, and when the number of items is saturated, consumers really want to buy cultural and creative products with practical value and appreciation value under the current popular concept of minimalism. To develop new cultural and creative products that can be continuously kept and used, the integration of traditional arts and crafts may be the most effective. If the materials are upgraded and traditional arts and crafts techniques such as cloisonné, lacquer and silk-pinching are used (cloisonné is not only used on bracelets, but also on products such as chair armrests or lamps), the artistic value of the product is not only improved, but also can be collected and inherited in addition to continuous use, and the service life of the product is prolonged. The cultural and creative products will be more effective in history, or in the sustainable development. In the end, the cultural and creative products become the art works of sustainable appreciation and use. If the cultural and creative products want to have a long-term concept of design practicality and appreciation, the use function is the core [2].

\section{TECHNOLOGY GIVING NEW VALUES TO CULTURAL AND CREATIVE PRODUCTS}

The comprehensive utilization of traditional handicrafts and modern science and technology requires cross-border thinking. The use of scientific and technological means will make it easier to produce design effects. Integration with science and technology is the internal driving force for the sustainable development of product design. (Figure 4.)

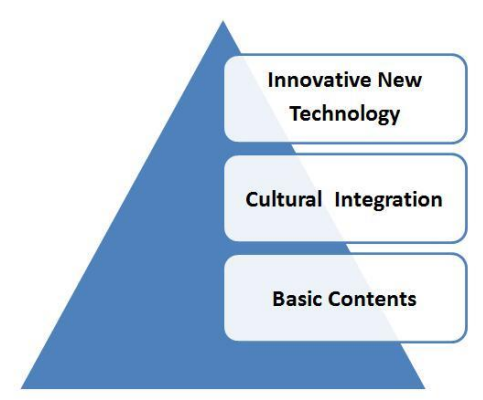

Figure 4 Layered Design of Cultural and Creative Products

\subsection{Technicalization of Design Means}

In the information and digital age, great changes have taken place in design method, and intelligent design methods have become a trend.The software designer must through intelligent design,define and satisfy the project's requirements. Virtual Reality (VR) can realize the conceived concept of cultural and creative product design in the virtual environment, and the design with good effect will eventually be put into the market. The computer monitors and improves the use, function, index and other parameters of the product, so that the user experience can be improved and the product development can be more scientific. With the support of technology, the speed of design of cultural and creative products has also increased dramatically. Intelligent design,virtual design and $3 \mathrm{D}$ printing have changed the slow pace of cultural and creative product design and made it highly efficient [3].

\subsection{The Use of New Materials}

Weaving is an antique technical skill, which 
continues the traditional techniques. Traditional materials are developing rapidly and the choice of materials is diverse. Especially the arts and crafts are mostly from the material acquisition of nature. With the support of new technologies, raw materials are optimized by modern means. The new processing technology enables these materials to obtain higher toughness and strength, and obtain more durable vitality. For example, the current Chinese weaving process uses special layers to reinforce rattan and bamboo or uses new chemical fiber materials, and even some waste materials can continue to be processed into products after layer treatment [4]. Although industrial production has replaced manual labor as the mainstream, and improvements in materials have led to simpler and more modern design language in cultural and creative products, the ancient traditional techniques still exude a unique humanistic beauty that cannot be replicated by machines today [5]. In the design of products such as chair weaving, tapestries and soft furnishings, the traditional art of weaving is almost inseparable. With the advancement of technology, the quality of cultural and creative products has been enhanced.

\section{BUILDING BRAND TO ENHANCE THE COMPETITIVENESS OF CULTURAL AND CREATIVE PRODUCTS}

\subsection{Standardizing the Design Process}

Cultural and creative products need brand to enhance product competitiveness. From the perspective of the commercial sales characteristics of cultural and creative products, the first priority is to analyze the audience and understand why they will buy them. The second is to have a clear design positioning; to form their own style positioning after the analysis of the style of similar products; to design classic models and series models; to have brand awareness, for example, the creation of IP can carry out brand promotion, and to use the brand communication method to enhance the visibility of we-media and expand the profit mode. Moreover, designers should pay attention to traditional arts and crafts, analyze the unique artistic characteristics of arts and crafts, get design inspiration from traditional arts and crafts to design modern cultural and creative products with different shapes and techniques, and enrich the design of cultural and creative products. Enterprises should establish relevant rules and process for operation, bringing good brand effect.

\subsection{Diversification of Brand Promotion Means}

SWOT analysis should be conducted first (Figure 5.) to carry out research and analysis on strengths, weaknesses, opportunities and threats. Marketing means should not be too single. Marketing methods can be used in the form of experience, precise targeting, necessary feedback and timely optimization of products, so as to maintain the brand image of cultural and creative products, do a good job of brand management, and find out their core strengths. Based on this, brand shall be positioned to confirm their own development direction. (Figure 6.) At present, the homogenization of cultural and creative product design in the market is serious, and the marketing methods of tourism cultural and creative products are almost the same everywhere. To establish an integrated marketing concept, considering product, price, channel, promotion factors, as well as consumer and other factors, process technology is one of the more important factors in the design of cultural and creative products. Integrating local arts and crafts technology is tantamount to adding the unique culture of the region, and the promotion of distinctive cultural products will have unique marketing points and real vitality.

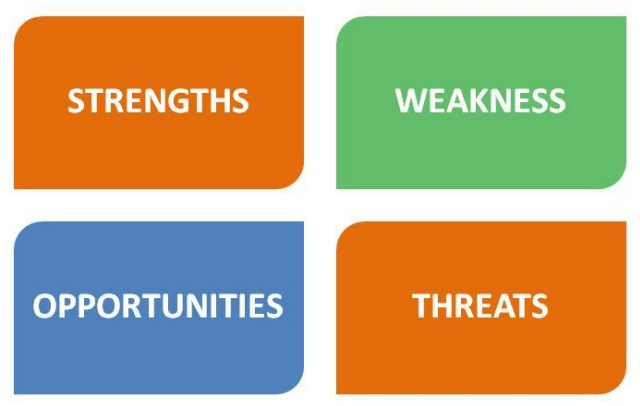

1. Whether the product is novel

2. Whether the manufacturing process is complicated

3. Whether market channels are unblocked

4. Whether there is an advantage in price

Figure 5 SWOT Analysis of the Product

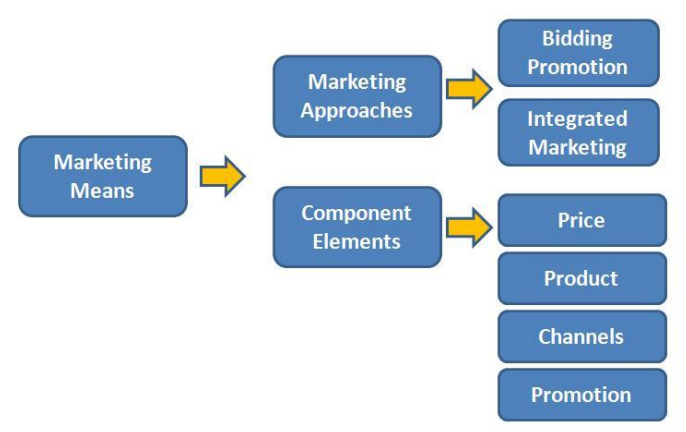

Figure 6 Marketing Approaches and Element Composition 


\subsection{Undertaking Social Responsibility to Enhance Brand Value}

Brand undertaking social responsibility can effectively establish and enhance brand image. A good brand cannot be limited to its own profit interests, but it is necessary to make use of the influence of its own brand to promote the culture of the region, enhance the brand value of the region and contribute to the regional economy. It makes the audience have a psychological and emotional sense of mutual identity and trust for its brand, and also helps to deepen the audience's understanding of the brand and their goodwill.

Traditional crafts need to be inherited, not simply as a copy of the original craft, but to be integrated into modern design to become an inherited media body. Arts and crafts can also be continued, promoted and inherited. We should build an innovative development system and bring the value of handicrafts back into play. It is known that traditional arts and crafts are now in urgent need of new media for sustainable development. For example, on the one hand, some of the non-heritage handicrafts have been difficult to get effective inheritance and protection among modern culture; and on the other hand, the cultural and creative products lack uniqueness and characteristics, so the combination of both is the inevitable path. In the general environment diagram of the project organization, we can clearly see the impact of the product project on the society, while various changes in the macro environment affect the project in turn. Incorporating handicrafts or arts and crafts is original design, and the charm of arts and crafts of various regions is increasingly shown in front of people's eyes. Traditional arts and crafts actually have a deep cultural and historical heritage. How corporate social responsibility is applied and shown in contemporary cultural and creative product design is an issue that requires serious consideration and research by designers today. (Figure7.) The quality of craftsmanship and innovation should always be placed in the design. The perfect combination of aesthetic value and quality in product design should make the product more brand competitive. The overall study and vision of the development strategy of cultural and creative product design should be launched, and corresponding solutions and development strategies should be proposed to explore the development and brand building of cultural and creative products in the future market [6].

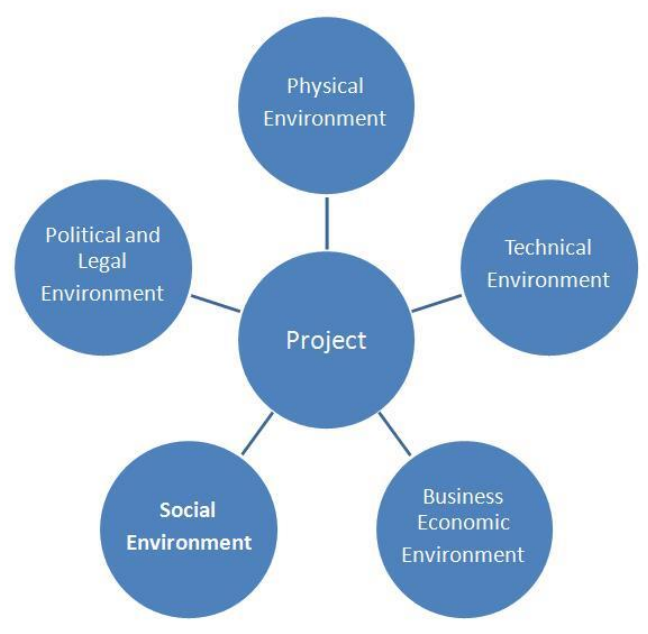

Figure 7 General Environment of the Project Organization

\section{CONCLUSIONS}

Only local design can lead modern Chinese design to the forefront of the world, both inheriting cultural traditions and promoting products. Based on the development of industrialization at the moment, many products also need personality and even more upgrading. The integration of arts and crafts can solve these problems. The combination of traditional arts and crafts as valuable cultural heritage inheritance and modern cultural and creative product design is rich in connotation, including the inheritance of fine culture, the spiritual quality of artisans, and innovative design concepts. The combination of the two provides a guarantee for the research and development of cultural and creative products, and provides a driving force for the sustainable development of arts and crafts and cultural and creative products.

\section{REFERENCES}

[1] Kim, H.J. (2010) Evaluation of Color Application of Homepage of Domestic and Foreign Online Bookstores-Focused on the Quantitative Evaluation of Aesthetic Measure and Cross-national Comparative Analysis-. Journal of Digital Design,163-174.

[2] Guo, S; Choi, T-M; Shen, B. (2020) Green product development under competition: A study of the fashion apparel industry. European Journal of Operational Research.523-538

[3] Kermavnar T; Shannon A; O’Sullivan L W. (2021) The application of additive manufacturing / 3D printing in ergonomic aspects of product design: A systematic review.97:103528 
[4] Romani A; Rognoli V; Levi M (2021) Design, Materials, and Extrusion-Based Additive Manufacturing in Circular Economy Contexts: From Waste to New Products, 7269.

[5] Kmita.A. (2018)Decorative patterns and sets of colors inspired by folk and applied arts of upper silesia, a popularization of visual cultural heritage of the region.Color Research \& Application, 942-950.

[6] Zhang $Z$ et al. (2007) Project organization and communication, In:XiujuanLu.Project Management, China Science Publishing \& Media,Beijing.pp. 264-265. 